OPEN ACCESS

Edited by:

Miguel Angel Prieto Lage,

University of Vigo, Spain

Reviewed by:

Javier E. Alvarez,

University of Vigo, Spain

Antía González Pereira,

University of Vigo, Spain

Pascual García-Pérez,

University of Vigo, Spain

*Correspondence:

Valentina Parra

vparra@ciq.uchile.cl

Rodrigo Troncoso

rtroncoso@inta.uchile.c

Specialty section:

This article was submitted to

Food Chemistry,

a section of the journal

Frontiers in Nutrition

Received: 13 September 2021

Accepted: 15 October 2021

Published: 12 November 2021

Citation:

Eynaudi A, Díaz-Castro F, Bórquez JC,

Bravo-Sagua $R$, Parra $V$ and

Troncoso R (2021) Differential Effects

of Oleic and Palmitic Acids on Lipid

Droplet-Mitochondria Interaction in the

Hepatic Cell Line HepG2.

Front. Nutr. 8:775382

doi: 10.3389/fnut.2021.775382

\section{Differential Effects of Oleic and Palmitic Acids on Lipid Droplet-Mitochondria Interaction in
the Hepatic Cell Line HepG2}

Andrea Eynaudi ${ }^{1}$, Francisco Díaz-Castro ${ }^{1}$, Juan Carlos Bórquez ${ }^{1}$, Roberto Bravo-Sagua ${ }^{2,3,4}$, Valentina Parra ${ }^{3,5 *}$ and Rodrigo Troncoso ${ }^{1,3 *}$

1 Laboratorio de Investigación en Nutrición y Actividad Física (LABINAF), Instituto de Nutrición y Tecnología de los Alimentos (INTA), Universidad de Chile, Santiago, Chile, ${ }^{2}$ Laboratorio de Obesidad y Metabolismo Energético (OMEGA), Instituto de Nutrición y Tecnología de los Alimentos (INTA), Universidad de Chile, Santiago, Chile, ${ }^{3}$ Advanced Center for Chronic Diseases (ACCDiS), Facultad de Ciencias Químicas y Farmacéuticas, Universidad de Chile, Santiago, Chile, ${ }^{4}$ Red de Investigación en Envejecimiento Saludable, Consorcio de Universidades del Estado de Chile, Santiago, Chile, ${ }^{5}$ Red Para el Estudio de Enfermedades Cardiopulmonares de Alta Letalidad (REECPAL), Universidad de Chile, Santiago, Chile

Fatty acid overload, either of the saturated palmitic acid (PA) or the unsaturated oleic acid $(\mathrm{OA})$, causes triglyceride accumulation into specialized organelles termed lipid droplets (LD). However, only PA overload leads to liver damage mediated by mitochondrial dysfunction. Whether these divergent outcomes stem from differential effects of PA and OA on LD and mitochondria joint dynamics remains to be uncovered. Here, we contrast how both fatty acids impact the morphology and interaction between both organelles and mitochondrial bioenergetics in HepG2 cells. Using confocal microscopy, we showed that short-term (2-24h) OA overload promotes more and bigger LD accumulation than PA. Oxygen polarography indicated that both treatments stimulated mitochondrial respiration; however, OA favored an overall build-up of the mitochondrial potential, and PA evoked mitochondrial fragmentation, concomitant with an ATP-oriented metabolism. Even though PA-induced a lesser increase in LD-mitochondria proximity than OA, those LD associated with highly active mitochondria suggest that they interact mainly to fuel fatty acid oxidation and ATP synthesis (that is, metabolically "active" LD). On the contrary, OA overload seemingly stimulated LD-mitochondria interaction mainly for LD growth (thus metabolically "passive" LDs). In sum, these differences point out that OA readily accumulates in LD, likely reducing their toxicity, while PA preferably stimulates mitochondrial oxidative metabolism, which may contribute to liver damage progression.

Keywords: lipid droplets, mitochondria, fatty acids, hepatocytes, oxygen consumption 


\section{INTRODUCTION}

Non-alcoholic fatty liver disease (NAFLD) is the most common liver disease, affecting $15-30 \%$ of the world population. It has a direct association with obesity, insulin resistance, and cardiovascular diseases (1) and characterizes by excessive fat accumulation in the liver ( $>5 \%$ in hepatocytes) (2). Therefore, its development tightly relates to fatty acid (FA) intake. On this regard, evidence supports the idea that saturated FA predisposes to hepatic lipid accumulation (termed steatosis), while unsaturated FA could be protective (3).

The most abundant saturated and monounsaturated FA in the Western diet are palmitic (PA, C16:0) and oleic acid (OA, C18:1 n-9) (4). During intestinal absorption, they are esterified into triglycerides (TG), and then delivered to the liver, which subsequently distributes them to other organs. However, excessive levels of either PA and OA lead to steatosis but with distinct cellular outcomes $(5,6)$. On the one hand, PA treatment causes liver lipotoxicity via oxidative stress, resulting in endoplasmic reticulum (ER) and mitochondrial dysfunction, and ultimately cell demise (5-7). In contrast, primary cultures of mouse hepatocytes treated with OA do not display either increased generation of oxygen radicals or signs of mitochondrial dysfunction or apoptosis. Moreover, in the hepatocyte-derived cell line HepG2, OA even prevented PA-induced liver lipotoxicity $(6,7)$.

Acting as protection against lipotoxicity, lipid droplets (LD) serve as TG deposits, thereby preventing fat accumulation in other cell compartments (8). Structurally, they comprise a nucleus of neutral lipids, mostly TG, surrounded by a monolayer of phospholipids and specific coating proteins, such as those belonging to the perilipin (PLIN) family. LD are highly dynamic organelles, varying in number and size according to storage requirements. Conversely, $\mathrm{LD}$ also hydrolyze TG, thus releasing free fatty acids, which serve as energy sources through their degradation (9). Fatty acid degradation takes place at mitochondria, which produce ATP through oxygen-driven oxidation (10). Like LDs, mitochondria vary in number and size to cope with varying nutritional scenarios, such as fasting and physical activity (11). In this sense, studies suggest that smaller mitochondria are more oxidative and thus, synthesize ATP more efficiently (8).

Apart from individual dynamics, mounting evidence shows that mitochondria and LD physically interact, especially in tissues with a high capacity for fatty acid oxidation and storage, such as the liver, heart, brown adipose tissue, and skeletal muscle (8, 12). For instance, PLIN5 mediates LD-mitochondrial interaction in the mouse liver cell line AML12 (13) and cardiac tissue

\footnotetext{
Abbreviations: ATP, Adenosine triphosphate; BSA, Fetal bovine serum; ER, Endoplasmic reticulum; ETC, Electron transport chain; FA, Fatty acids; FCCP, Carbonyl cyanide 4-(trifluoromethoxy)phenylhydrazone; GAPDH, Glyceraldehyde-3-phosphate dehydrogenase; LD, Lipid droplets; MFN2, Mitofusin 2; MIGA2, Mitoguardin 2; NAFLD, Non-alcoholic fatty liver disease; OA, Oleic acid; OCR, Oxygen consumption rate; OPA1, OPA1 mitochondrial dynamin-like GTPase; PA, Palmitic acid; PBS, Phosphate-buffered saline; PLIN, Perilipin family of proteins; PLIN1, Perilipin 1; PLIN2, Perilipin 2; PLIN5, Perilipin 5; TG, Triglycerides; $\Delta \psi \mathrm{m}$, Mitochondrial transmembrane potential.
}

of mice, resulting in LD expansion and a decrease in fatty acid oxidation (14). Likewise, in adipose tissue, PLIN2 binds the mitochondrial protein MIGA2, thus bridging mitochondria and LD (15). Furthermore, PLIN5 and PLIN2 form a complex, which favors mitochondrial recruitment to the surface of LDs in cardiomyocytes (14). In adipose tissue, PLIN1 reportedly promotes LD interaction with mitochondria by binding to the proteins MFN2 and OPA1 at the mitochondrial surface $(16,17)$. Interestingly, Benador et al. attained similar results about LD-mitochondria association in brown adipose tissue (8). In hepatocytes, the physiological role of LD-mitochondria interaction is yet to be unveiled. Also, despite being the most abundant dietary fatty acids, little is known about the differences between the effect of PA and OA on LD morphology and contact with mitochondria in hepatocytes (Figure 1A). Therefore, the objective of this work was to contrast how PA and OA impact LDmitochondria dynamics and mitochondrial bioenergetics and how these processes are associated with the development of hepatic steatosis.

\section{METHODS}

\section{Cell Culture and Treatments}

The HepG2 cell line was obtained from the American Type Culture Collection (ATCC HB-8065). Cells were cultured in Dulbecco's modified Eagle's medium (DMEM 11965-09; Thermo Fisher Scientific), supplemented with 10\% fetal bovine serum (FBS), $1 \%$ penicillin-streptomycin antibiotics (Biological Industries) and maintained at $37^{\circ} \mathrm{C}$ with a $5 \% \mathrm{CO}_{2}$ atmosphere. Hepatocytes were exposed to $200 \mu \mathrm{M}$ OA (P1383, SigmaAldrich) or $200 \mu \mathrm{M}$ PA (P0500; Sigma-Aldrich) conjugated to fatty acid-free bovine serum albumin (BSA) (A8806; SigmaAldrich) for 2, 6, 18 and $24 \mathrm{~h}$, as previously described (18). Cells treated with BSA were used as controls.

\section{Western Blot Analysis}

Cell protein extracts were obtained using T-PER lysis buffer (78510, Thermo Fisher Scientific), supplemented with protease (\#04693159001, Roche) and phosphatase (\#04906845001, Roche) inhibitors. Homogenates were centrifuged at $14,500 \mathrm{G}$ for $10 \mathrm{~min}$, and the supernatants were collected for measuring protein concentrations with the BCA protein assay (23227, Thermo Fisher Scientific). Protein extracts of $50 \mu \mathrm{g}$ were resolved in 10\% SDS-polyacrylamide gels, transferred onto PVDF membranes previously activated with methanol, and blotted overnight with primary antibodies at $4{ }^{\circ} \mathrm{C}$. The antibodies and dilutions were: anti-PLIN2 (1:1000; \#MA5-24797, Thermo Fisher Scientific), anti-PLIN5 (1:1000; \#GP31, Progen), and anti-MFN2 (1:1000; \#ab50838, Abcam). The membranes were then incubated for $1 \mathrm{~h}$ with the following secondary HRP-coupled antibodies: antimouse (1:5000; \#402335, Calbiochem), anti-guinea pig (1:5000; \#ab6908, Abcam) or anti-rabbit (1:5000; \#401315, Calbiochem) and revealed with the EZ-ECL detection kit (DW1029, Biological Industries). A C-DiGit $\AA$ Blot Scanner and the Image Studio software version 3.1 (LI-COR) were used for image acquisition and densitometric analysis. GAPDH (1:5000; \#MAB374, SigmaAldrich) was used as a loading control. 

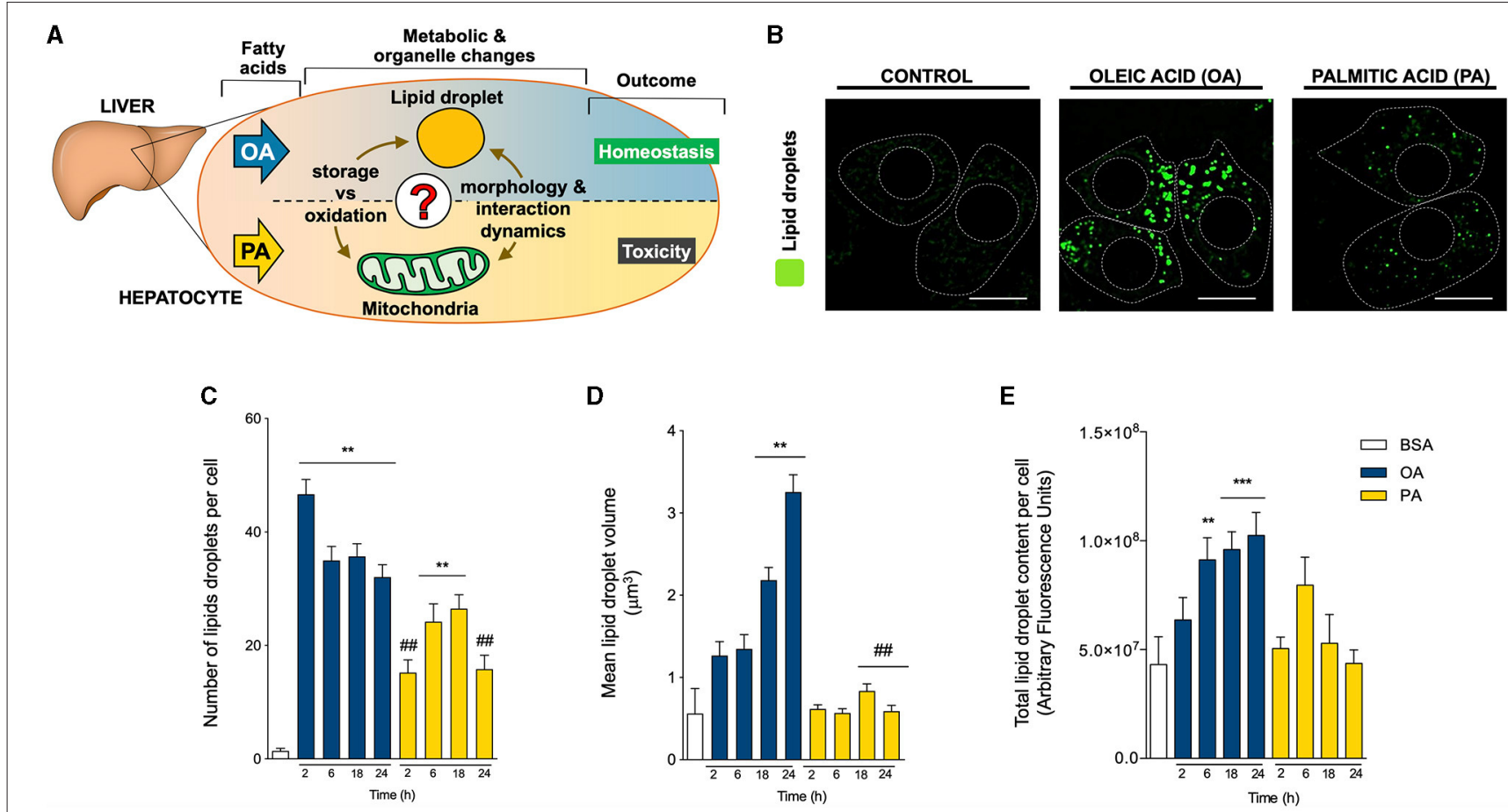

FIGURE 1 | Oleic and palmitic acids differentially remodel lipid droplet morphology in HepG2 cells. (A) In the liver, oleic acid (OA) overload seemingly does not damage hepatocyte function, while palmitic acid (PA) is evidently toxic. To date, the underlying mechanism of this difference remains unknown in terms of fatty acid metabolism and mitochondria-lipid droplet interactions and morphology. (B) Representative confocal imaging of HepG2 cells treated with $200 \mu \mathrm{M}$ of OA or PA during $18 \mathrm{~h}$ and stained with Bodipy 493/503 (green) for lipid droplet (LD) visualization. Segmented lines represent the cellular and nuclear contours. Scale bar: $10 \mu \mathrm{m}$. (C) Quantification of the number of LDs per cell in images treated as in A for 0-24 h. (D) Mean individual volume of LDs in HepG2 cells treated as in A for 0-24 h. (E) Quantification of the total Bodipy 493/503 fluorescence in HepG2 cells treated as in A for 0-24 h. Data are from $N=6-20$ cells analyzed from three independent experiments. Results are shown as mean \pm SEM; ${ }^{\star} p<0.05 ;{ }^{\star \star} p<0.01$; ${ }^{\star \star \star} p<0.001$ vs. BSA; $\# p<0.05$; and $\# \# p<0.01$ vs. OA at the same time conditions.

\section{ATP Measurements}

Intracellular ATP content was determined using the luciferin/luciferase-based ATP detection kit CellTiter-Glo Luminescent Cell Viability Assay (Promega) following the manufacturer's instructions as described in (19). Briefly, HepG2 cells were cultured in 96-well Petri dishes and washed 3 times with PBS before incubation with the reagent. Sample luminescence was quantified in a Synergy 2 microplate reader (BioTek Instruments). Data were normalized as fold of changes over control. Treatment with oligomycin $(5 \mu \mathrm{g} / \mathrm{mL})$ for $3 \mathrm{~h}$ was used as a negative control.

\section{Oxygen Consumption}

Cells were seeded in $60 \mathrm{~mm}$ Petri dishes at $80 \%$ confluence and treated according to the experiment. After the different treatments, measurements were performed as previously described (20-22). In brief, cells were washed with PBS, trypsinized for $3 \mathrm{~min}$, and centrifuged at $200 \mathrm{G}$ for $5 \mathrm{~min}$. Then, the cells were resuspended in PBS and placed in the chamber of a Clark electrode (Oxygraph Plus, Hansatech). Basal respiration, proton leak, or ATPunlinked respiration (Oligomycin, $400 \mu \mathrm{M}$ ) and uncoupled respiration (FCCP $20 \mu \mathrm{M}$ ) were measured sequentially for 3 min. Data obtained were standardized to basal control respiration.

\section{Microscopy}

Cells were plated on $12 \mathrm{~mm}$ Petri dishes and treated according to the experiment. To label LDs and mitochondria, cells were stained with BODIPY 493/503 (2 $\mu$ M; D3922, Invitrogen) and MitoTracker Orange (400 nM; M7510, Invitrogen) for $25 \mathrm{~min}$ at $37{ }^{\circ} \mathrm{C}$. Then, the cells were fixed with $4 \%$ paraformaldehyde and $0.01 \%$ Hoechst in PBS, and mounted in Dako Fluorescence Mounting Medium (S3023, DakoAgilent). Fixed cells were imaged using a Nikon C2 PlusSiR confocal microscope. 6-20 cells were registered for 3 independent experiments.

\section{Image Analysis}

Images acquired were deconvoluted, background-subtracted, thresholded, and analyzed with ImageJ software (NIH). LDs and mitochondria number and individual volume were quantified using the 3D Object Counter plugin, as previously described (20-23). LD-mitochondria colocalization was determined within one focal plane using the JACoP plugin $(20,22,23)$. The mitochondrial potential was defined in relation to MitoTracker Orange fluorescence intensity, and was analyzed within a single 


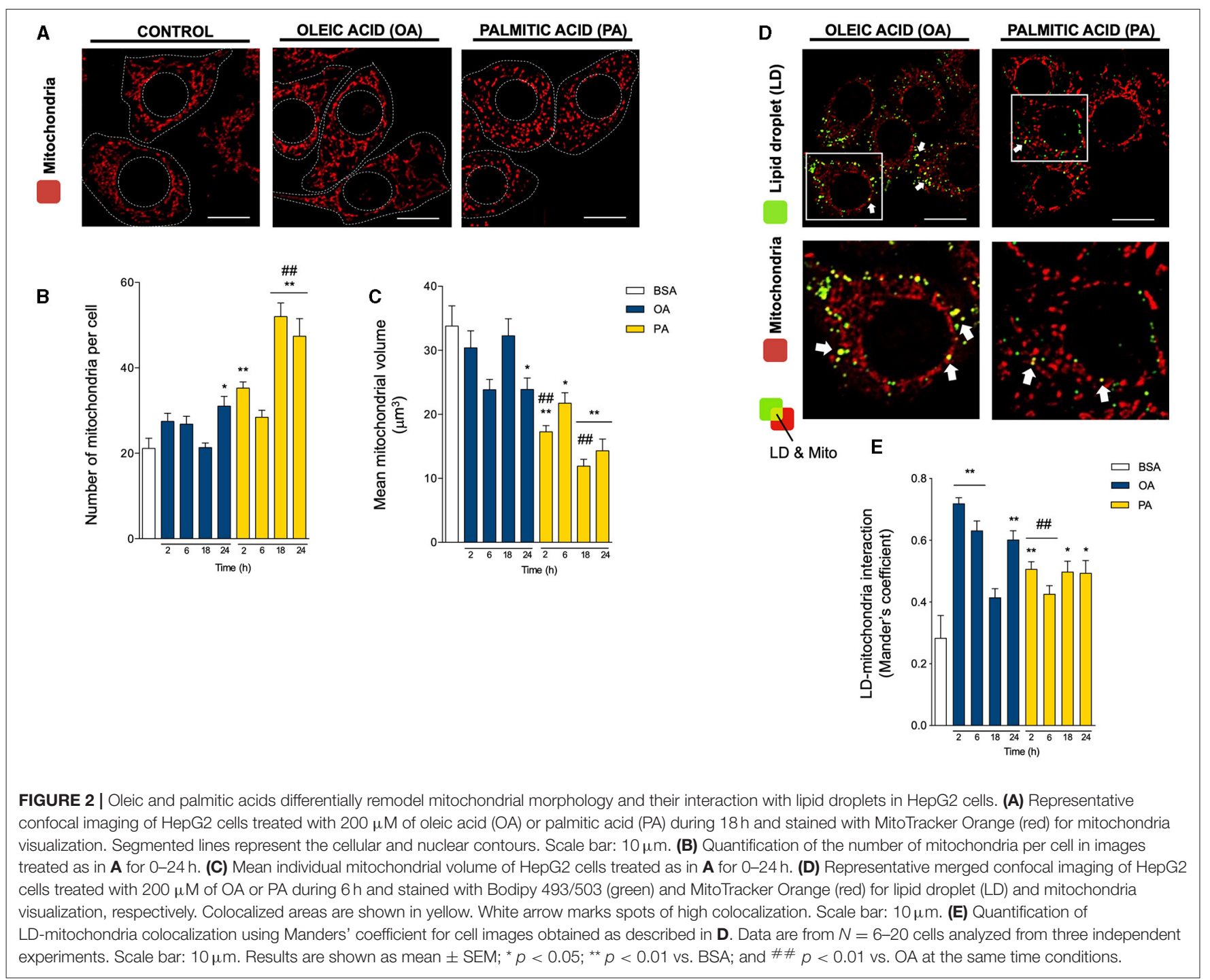

plane at the cell equator with the Analyze Particles function (20, 22). The mitochondrial potential of bound LD and non-boundLD was performed by constructing a compartment consisting of $10 \mathrm{z}$-planes of the sites where mitochondria colocalize with LDs. Then, the intersected or excluded mitochondrial fluorescence of bound LD and non-bound-LD was quantified, respectively. Image intersections were obtained using the Image Calculator command of ImageJ (“AND” operator) (22).

\section{Statistical Analysis}

All statistical analyses were performed using GraphPad Prism software, version 6 (San Diego, CA, USA). Data are expressed as mean \pm SEM of at least three independent experiments. Data were analyzed by one-way ANOVA, and, when appropriate, comparisons between groups were performed using Tukey's or Dunnett's post-hoc tests. A two-tailed Pearson's coefficient was used for correlation analysis. Differences were considered significant at $P<0.05$ (that is, a confidence level of $95 \%, \alpha=0.05)$.

\section{RESULTS}

\section{Oleic and Palmitic Acids Have Differential Effects on LD and Mitochondrial Dynamics}

To evaluate whether OA or PA affects the morphology of LDs, HepG2 hepatocytes were treated with OA or PA between $0-24 \mathrm{~h}$. Whereas OA (Figure 1B) stimulated the appearance of big and bright droplets in hepatocytes, mainly in the central region of the HepG2 cells, PA triggered the emergence of evenly distributed smaller droplets. To quantify this effect, LD morphology was also assessed according to the number and volume of individual isolated elements through $3 \mathrm{D}$ reconstitution of confocal stacks (20-23) and the total fluorescence of BODIPY. Compared to BSA, OA significantly increased the number of LDs per cell at all the times analyzed, reaching a $\sim 30$-fold increase as early as $2 \mathrm{~h}$ after treatment (Figure 1C). Subsequently, OA also led to a gradual increase in the volume of the LDs, reaching $\sim 4$ and $\sim 6$-fold, compared to controls after 18 and $24 \mathrm{~h}$, respectively (Figure 1D). In terms of total BODIPY fluorescence, only OA 
triggered a significant increase in the fluorescence after $6 \mathrm{~h}$ of treatment; moreover, this increase was maintained at later times of 18 and $24 \mathrm{~h}$ (Figure 1E). These results agree with the rapid nucleation of new LDs, which later grow over time. On the other hand, treatment with PA led to a slower increase in the number of LD per cell, which was significant ( $\sim 20$-fold) at 6 and $18 \mathrm{~h}$ of treatment (Figure 1C). In contrast to OA, PA did not change the volume of the LDs, suggesting slow nucleation of new LD without a growing phase (Figure 1D).

We next evaluated mitochondrial morphology in HepG2 hepatocytes using the mitochondrial-specific MitoTracker Orange probe $(400 \mathrm{nM}, 25 \mathrm{~min})$ and 3D-reconstruction imaging (Figures 2A-C), as we have previously reported $(21,23)$. In contrast to the steep changes in LD morphology, OA only caused a slight but significant increase in the number of mitochondria per cell, concomitant with a decrease in their volume after $24 \mathrm{~h}$, indicative of the induction of mitochondrial fission (Figures 2B,C). Meanwhile, PA treatment triggered a faster and more intense process of mitochondrial fragmentation, noticeable after $2 \mathrm{~h}$, reaching a $\sim 2$-fold increase in the number of mitochondria per cell and a significant $\sim 2$-fold reduction in mitochondrial volume (Figures 2B,C). The magnitude of these differences reveals maintenance in the total size of the mitochondrial network, mainly suggesting changes in the mitochondrial fusion/fission equilibrium. Our results with PA are consistent with their reported effects on mitochondrial morphology in other cell types (24-26).

To evaluate whether the changes in organelle dynamics affect their physical coupling, we then assessed LD-mitochondria proximity, evaluated as Mander's colocalization coefficients between LD and mitochondria (20, 22, 27) (Figures 2D,E). Both fatty acids increased LD-mitochondria proximity starting at $2 \mathrm{~h}$. However, OA treatment induced a significantly higher increase at $2 \mathrm{~h}$ (>2-fold), which later decreased to values similar to PA ( 1.5-fold). Taken together, these results suggest that OA treatment triggers the early appearance of more and bigger LDs than PA, which are in closer contact with mitochondria in HepG2 hepatocytes.

\section{Oleic and Palmitic Acids Evoke Different Profiles of LD-Mitochondria Interaction Proteins}

PLINs are the main structural proteins of LDs, and determine their structure, metabolism, and interaction with other organelles $(28,29)$. While PLIN2 is ubiquitously expressed, PLIN5 is especially enriched in tissues with high levels of mitochondrial oxidation, such as the liver (29). PLIN5 reportedly regulates mitochondrial recruitment to $\mathrm{LD}$, thereby regulating mitochondrial metabolism $(8,29,30)$. On the other hand, MFN2 not only promotes mitochondrial fusion and boosts mitochondrial metabolism $(31,32)$, but also binds to PLIN1 to facilitate the LD-mitochondria interaction in adipose tissue $(17,33)$. Due to their importance, we measured their relative abundance in our experimental model through Western blot analysis (Figure 3A). As shown in Figures 3A,B, PA triggered a significant acute increase in PLIN2 at $6 \mathrm{~h}$ of treatment, without altering MFN2 or PLIN5, compared to control. On the other hand, OA increased both PLIN5 and MFN2 (Figures 3A,C,D) at later times (18-24h). These results suggest that OA promotes PLIN5-MFN2-mediated LD-mitochondria interaction, while PA favors the presence of PLIN2 at the surface of LDs.

\section{Oleic and Palmitic Acids Differentially Modulate Mitochondrial Oxidative Function}

Mitochondria act as oxidative centers for different metabolites, such as fatty acids, which ultimately fuel a chain of redox reactions driven by oxygen-mediated oxidation. The protein complexes that catalyze this redox process (termed the electron transport chain, ETC) pump protons across the inner mitochondrial membrane. This creates an electrochemical gradient, which entails the generation of a mitochondrial transmembrane potential $(\Delta \psi \mathrm{m})$. The resulting proton-motive force drives ATP production by transporting the protons back across the mitochondrial inner membrane through the ATP synthase enzyme $(23,34)$. Of note, this mechanism implies that ATP production dissipates the $\Delta \psi \mathrm{m}$ (Figure 4A). Thus, to characterize the differential effects of OA and PA treatments on mitochondrial metabolism, we analyzed three defining parameters of mitochondrial bioenergetics (34): $\Delta \psi \mathrm{m}, \mathrm{O}_{2}$ consumption, and ATP levels.

We first evaluated the $\Delta \psi \mathrm{m}$ by using the mitochondriaspecific MitoTracker Orange potentiometric probe and confocal microscopy. Because PA treatment triggers mitochondrial fragmentation and a concomitant decrease in mitochondrial volume, to quantify the $\Delta \psi \mathrm{m}$, we evaluated both the mean mitochondrial fluorescence and the total fluorescence per cell of MitoTracker Orange. The first fluorescence parameter addresses the bioenergetic state of individual mitochondria, and the second parameter evaluates the metabolic state of the cell as a whole. Figures 4B,C shows that both $\mathrm{OA}$ and PA treatments increased the mean and total fluorescence levels of MitoTracker Orange, starting at $2 \mathrm{~h}$, indicating a boost in the $\Delta \psi \mathrm{m}$. In the case of OA, the $\Delta \psi \mathrm{m}$ peaked at $6 \mathrm{~h}$ and remained high for all the analyzed times. On the other hand, PA treatment led to a subsequent decrease in the $\Delta \psi \mathrm{m}$ to baseline levels after 6 and $18 \mathrm{~h}$, followed by a second rise in the $\Delta \psi \mathrm{m}$, noticeable at $24 \mathrm{~h}$, thus suggesting a two-phase response for this fatty acid.

Next, we assessed the $\mathrm{O}_{2}$ consumption rate (OCR) as a measure of mitochondrial oxidative activity. Treatments with OA or PA increased basal OCR but with different increment profiles (Figure 4D). In the case of OA, the increment was slower, becoming significant at $6 \mathrm{~h}$. PA triggered a faster increase in the OCR, as early as $2 \mathrm{~h}$, which continued at $6 \mathrm{~h}$ (Figure $4 \mathrm{D}$ ). We also measured OCR in the presence of oligomycin, an inhibitor of ATP synthesis, which reveals the amount of mitochondrial respiration not associated with ATP production (Figure 4D). Neither OA nor PA changed the OCR in the presence of oligomycin, thereby suggesting that neither treatment affects the mitochondrial coupling between respiration and ATP production. In other words, it appears that mitochondria remain similarly "efficient". Additionally, we also measured OCR in the presence of FCCP, which dissipates the proton gradient 


\section{A}

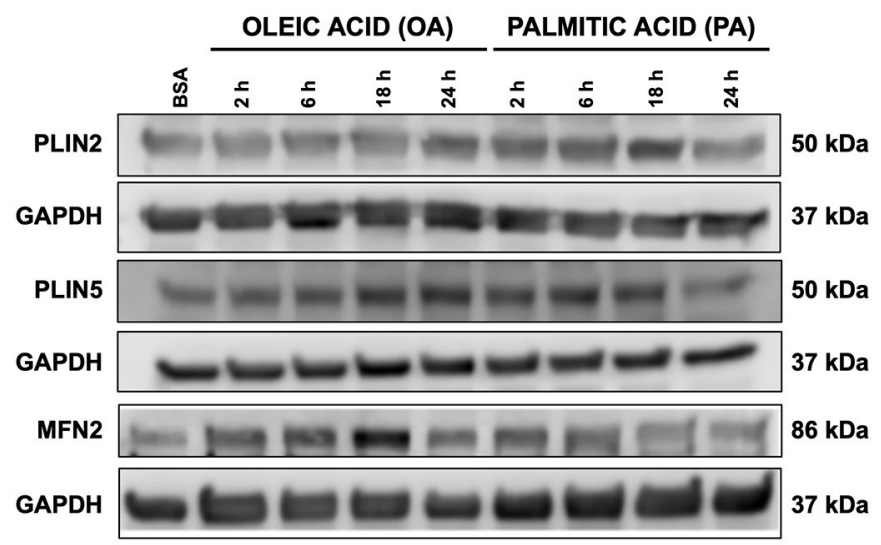

B

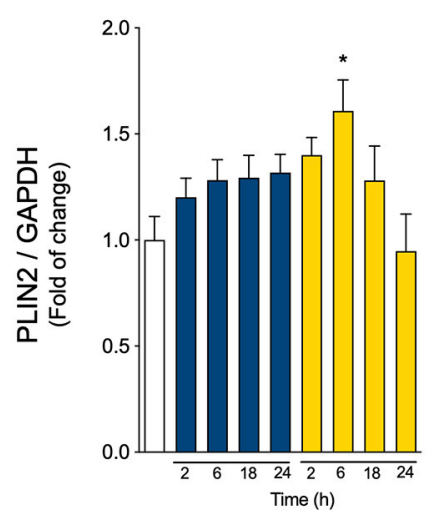

C

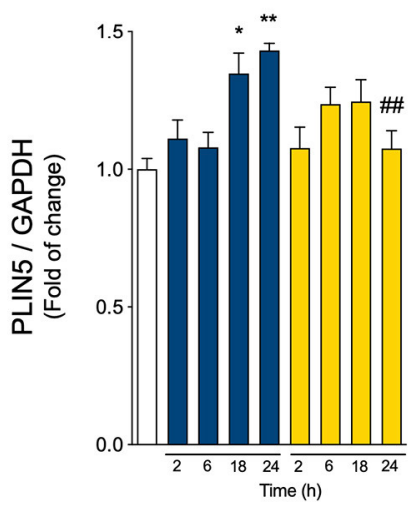

D

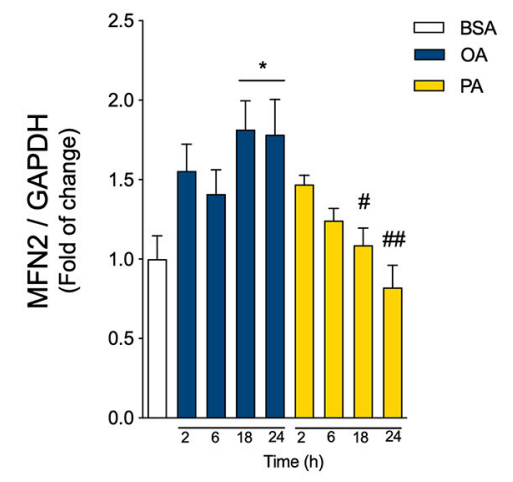

FIGURE 3 | Oleic and palmitic acids induce different patterns of lipid droplet-mitochondria tether proteins in HepG2 cells. (A) Representative Western blots of the proteins associated with lipid droplet (LD)-mitochondria physical interaction: Perilipin 2 and 5 (PLIN2, PLIN5), and Mitofusin 2 (MFN2) in HepG2 cells treated with 200 $\mu \mathrm{M}$ of oleic acid (OA) or palmitic acid (PA) for 0-24 h. GAPDH was used as a loading control. (B-D) Densitometric quantification of the proteins indicated in $\mathbf{A}$. Data are expressed as mean \pm SEM of $N=7$ for PLIN2 and $N=4$ for PLIN5 and MFN2. ${ }^{*} p<0.05 ;{ }^{* *} p<0.01$ vs. BSA; \# $p<0.05$; and $\# \#$ \# $p<01$ vs. OA at the same time conditions.

at the inner mitochondrial membrane (i.e., $\Delta \psi \mathrm{m}$ ). Given that mitochondrial respiration is a process that works against the proton gradient, the elimination of the $\Delta \psi \mathrm{m}$ allows respiration to reach its maximum levels. As with oligomycin, neither OA nor PA changed the magnitude of FCCP-induced OCR (Figure 4D), implying that mitochondria maintain a similar "maximal performance" across treatments. Taken together, these results suggest that the changes in basal OCR would not be due to differences in mitochondrial functional capacity (for example, increases in mitochondrial functional units or damage of existing ones), but rather to regulatory changes, such as differential substrate availability or upstream signaling cascades.

Similar to OCR, PA treatment triggered a significant increase in ATP production as soon as $2 \mathrm{~h}$, which remained high at least until $6 \mathrm{~h}$. However, OA treatment led to a slower ATP increase, which reached significance at $6 \mathrm{~h}$ (Figure 4E). As an operational indicator of the mitochondrial functional profiles, we used the relationship between ATP production and the $\Delta \psi \mathrm{m}$ (Figure 4F). At $2 \mathrm{~h}$, the $\mathrm{ATP} / \Delta \psi \mathrm{m}$ ratio remained relatively constant for both conditions. Nonetheless, at $6 \mathrm{~h}$ there was a divergence: the mitochondrial network from PA-treated cells appeared more ATP production-oriented, while the network from OAtreated cells seemed to prefer $\Delta \psi \mathrm{m}$ build-up. Altogether, these results suggest that both treatments stimulate mitochondrial bioenergetics, but with different functional profiles.

\section{Oleic and Palmitic Acids Stimulate Divergent Specialization of Mitochondrial Populations}

In sum, both PA and OA fatty acids induce distinct changes in mitochondrial morphology, LD-mitochondria interaction, and their bridging proteins and at the level of mitochondrial functionality. However, our previous studies have shown that the mitochondrial network and its interactions are highly heterogeneous $(22,23)$. Thus, the observed changes probably involve a subpopulation of mitochondria as a sort of "specialization mechanism". Moreover, mounting evidence underscores an association between mitochondrial size and 


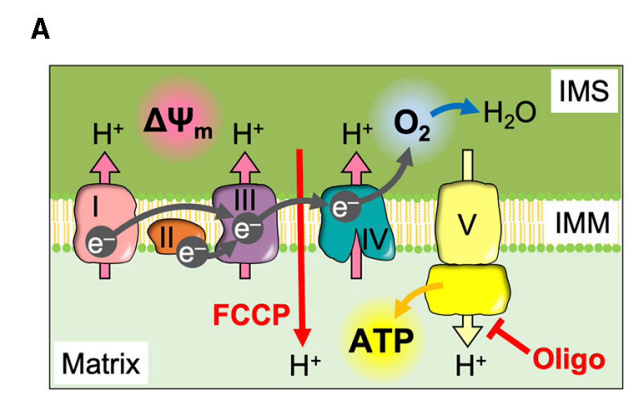

B

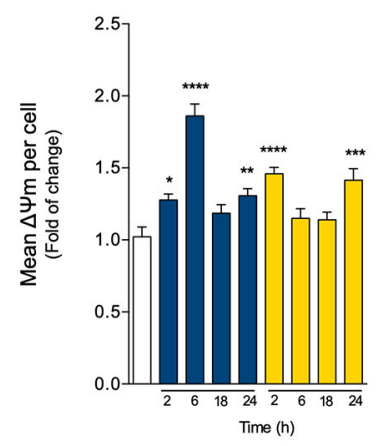

E

D

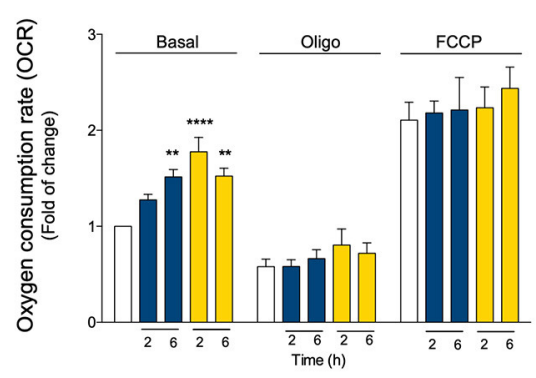

C

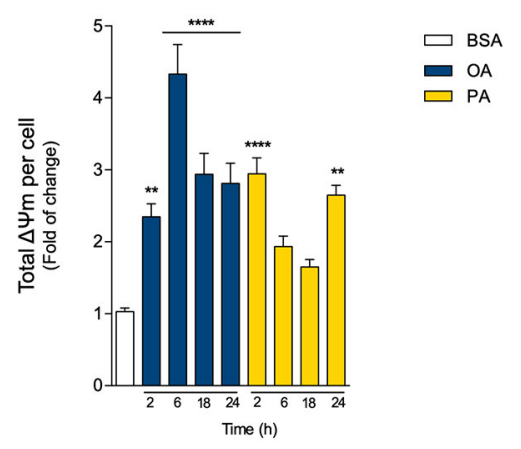

$\mathbf{F}$

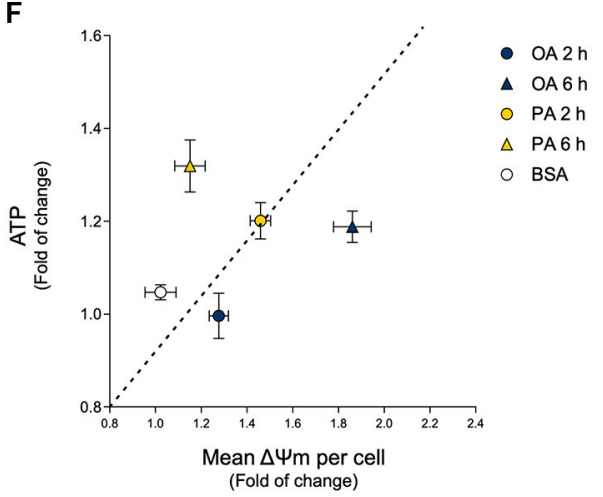

FIGURE 4 | Oleic and palmitic acids differentially modulate mitochondrial bioenergetics in HepG2 cells. (A) Schematics of mitochondrial respiration through the mitochondrial matrix and the intermembrane space (IMS). Complexes I, III and IV pump $\mathrm{H}^{+}$across the inner mitochondrial membrane (IMM), generating the mitochondrial transmembrane potential $(\Delta \Psi \mathrm{m})$, and ultimately, transforming $\mathrm{O}_{2}$ into $\mathrm{H}_{2} \mathrm{O}$. Then, complex $\mathrm{V}$ synthesizes ATP using the proton gradient of the $\Delta \Psi \mathrm{m}$. Oligomycin (Oligo) inhibits Complex V, thus leading to the build-up of the $\Delta \Psi \mathrm{m}$, which hampers mitochondrial respiration. FCCP, on the other hand, dissipates the $\Delta \Psi \mathrm{m}$, thereby eliminating the electrochemical restraint on mitochondrial respiration. (B,C) Mean and whole-cell $\Delta \psi \mathrm{m}$ of HepG2 cells treated with $200 \mu \mathrm{M}$ of oleic acid (OA) or palmitic acid (PA) for 0-24 h, measured using MitoTracker Orange and confocal fluorescence microscopy. Data are from $N=6-20$ cells analyzed from three independent experiments. (D) Oxygen consumption rate (OCR) of cells treated as in A, measured after 2 and $6 \mathrm{~h}$ of treatment. Baseline respiration, non-ATP-associated respiration (Oligo $400 \mu \mathrm{M}$ ), and the maximal respiratory capacity (FCCP $20 \mu \mathrm{M}$ ) were measured sequentially for 3 min ( $N=5$ ). (E) Intracellular ATP levels of cells treated as in $\mathbf{A}$, quantified after 2 and $6 \mathrm{~h}$ of treatment. Oligo $5 \mu \mathrm{g} / \mathrm{mL}$ for $3 \mathrm{~h}$ was used as a positive experimental control $(N=5)$. (F) Mitochondrial efficiency expressed as ATP levels vs. OCR at $2 \mathrm{~h}$ of OA or PA treatments. Results are shown as mean $\pm \mathrm{SEM} ;{ }^{*} p<0.05 ;{ }^{* \star} p<0.01 ;{ }^{* \star *} p<0.001$; and ${ }^{* \star * *} p<$ 0.0001 vs. BSA.

function, although in a cell type-dependent fashion (35). In brown adipocytes, which avidly oxidize FA, Benador et al. showed that LD-associated mitochondria are larger in size and mainly dedicated to ATP production and LD expansion, while smaller mitochondria associate mainly with FA oxidation (8). To address whether OA or PA replicate this behavior in our hepatocyte-derived cell line, we first compared the $\Delta \psi \mathrm{m}$ level of the LD-bound mitochondrial network with the rest of the mitochondrial network (hereafter termed "bulk mitochondria"). In Figures 5A,B, we show that although both $\mathrm{OA}$ and $\mathrm{PA}$ increase the $\Delta \psi \mathrm{m}$ of bulk mitochondria starting at $2 \mathrm{~h}$, only OA has a steady effect. In contrast, PA again displays a biphasic response, which faded at $6 \mathrm{~h}$, reappeared at $18 \mathrm{~h}$, and finally disappeared after $24 \mathrm{~h}$. In terms of LD-bound mitochondria, OA caused a minor accretion in their $\Delta \psi \mathrm{m}$, which became significant after $24 \mathrm{~h}$ of treatment (Figure 5C). On the other hand, PA augmented the $\Delta \psi \mathrm{m}$ after $6 \mathrm{~h}$, which faded at $18 \mathrm{~h}$, but reappeared at $24 \mathrm{~h}$. Curiously, the $\Delta \psi \mathrm{m}$ increments in bulk mitochondria interleaved with those of the LD-bound mitochondria. Taken together, these results suggest that $\mathrm{OA}$ induces steep nucleation and gradual expansion of LDs, which strongly interact with mitochondria exhibiting a relatively "weak" $\Delta \psi \mathrm{m}$. Contrarily, PA triggers the biogenesis of multiple but small LDs, which show a moderately increased interaction with mitochondria harboring a relatively "strong" $\Delta \psi \mathrm{m}$, associated with higher OCR and ATP production.

Finally, to assess the association between mitochondrial bioenergetics and LDs dynamics, we correlated the size of each individual $\mathrm{LD}$ with the $\Delta \psi \mathrm{m}$ of the associated mitochondria. We chose the temporality of $2 \mathrm{~h}$ because this is our earliest determination of LD nucleation, where the differences between $\mathrm{OA}$ and PA are most marked. In agreement with Figure 1, the histogram of Figure 5D shows that OA favors the emergence of larger LDs (cross-sectional area $>0.2 \mu \mathrm{m}^{2}$ ), while PA leads to the accumulation of smaller ones with smaller areas $(<0.2$ $\mu \mathrm{m}^{2}$ ). Then, we analyzed each LD from 18-20 cells in both 


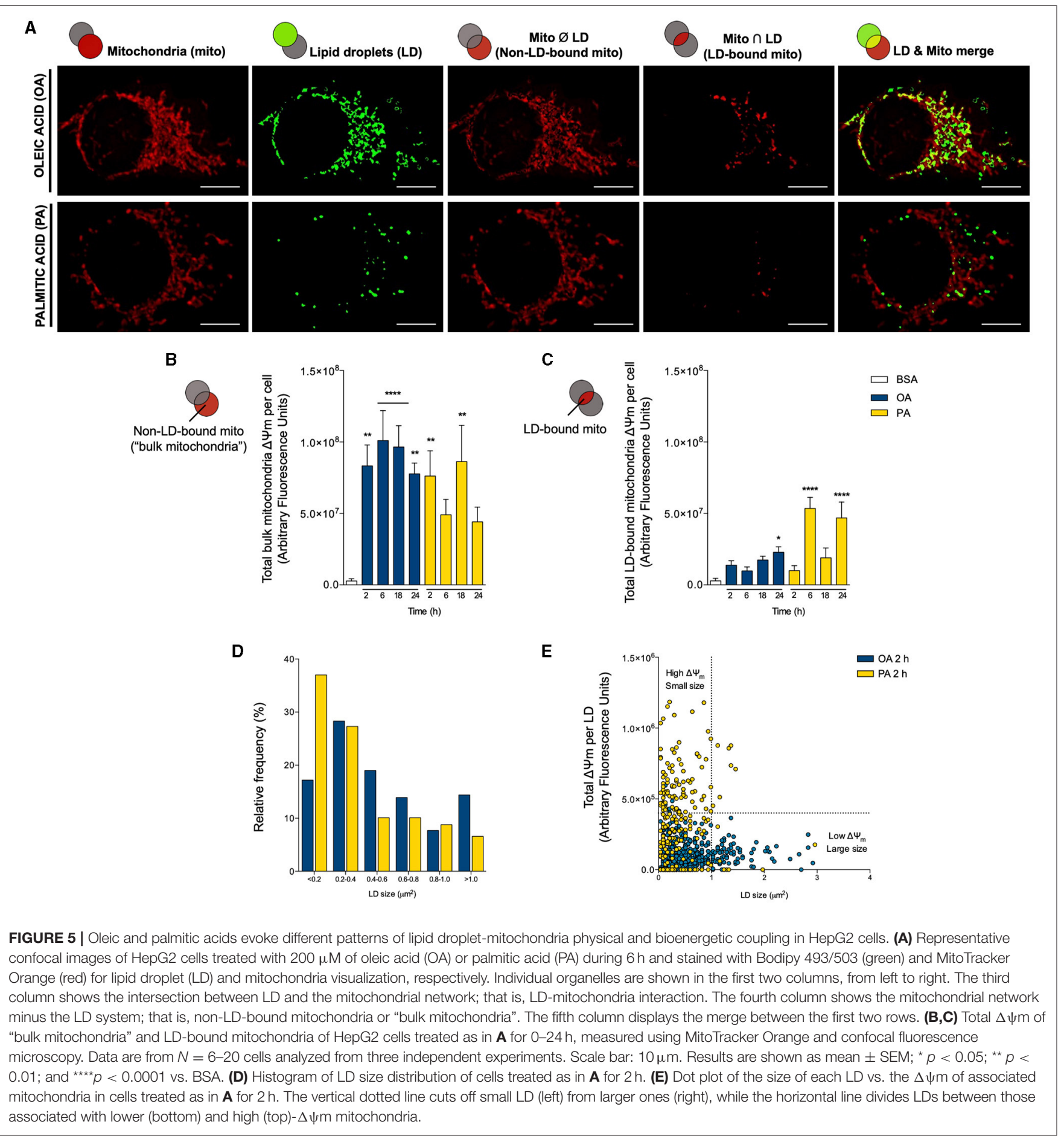

conditions and correlated the size of each LD with the associated total MitoTracker Orange fluorescence (Figure 5E). That is, we evaluated how active were the mitochondria associated with each LD (in terms of the $\Delta \psi \mathrm{m}$ ). We found that the different fatty acids promoted the appearance of distinct populations of LDs, respective to their size and the activity of the attached mitochondria. In either case, the "bulk LDs" consisted of units with smaller size $\left(<1 \mu \mathrm{m}^{2}\right)$, associated with mitochondria with lower $\Delta \psi \mathrm{m}$. As initially noted, OA led to the emergence of larger LDs, characterized by their colocalization with mitochondria with lower $\Delta \psi \mathrm{m}$. On the contrary, PA treatment did not promote the enlargement of LDs but triggered the appearance of a subpopulation of small-sized LDs associated with mitochondria with higher $\Delta \psi \mathrm{m}$. Altogether, these observations indicate that the 
unsaturated fatty acid OA promotes lipid accumulation in larger LDs proximal to mitochondria that build-up lower $\Delta \psi \mathrm{m}$ and that the saturated fatty acid PA favors smaller LDs in close apposition to mitochondria with higher $\Delta \psi \mathrm{m}$.

\section{DISCUSSION}

Within the cell, FA have structural and functional roles, and serve as an energy reservoir stored in the form of TG. The latter is essential to ensure a continuous FA supply, independent of external nutrient availability (36). However, high-fat diets associate with obesity and other related diseases (37), with several studies showing that the monounsaturated FA OA is less toxic than the saturated FA PA. Even more, OA can prevent PA-induced toxicity in hepatocytes (5-7), although the differential impact in LD-mitochondria dynamics and mitochondrial bioenergetics of each FA was not explored. Here, we report that PA and OA elicited a differential cellular response in the hepatocyte cell line HepG2. While both fatty acids led to massive TG accumulation, OA promoted the formation of more and bigger LDs, which were in closer contact with mitochondria compared to PA. Instead, the latter triggered a fast and intense process of mitochondrial fragmentation associated with increased OCR and ATP levels. Interestingly, the large LDs promoted by OA treatment were proximal to mitochondria with a baseline $\Delta \psi \mathrm{m}$ ("passive LDs"). In contrast, PA promoted the formation of small-sized LDs proximal to mitochondria with enhanced $\Delta \psi \mathrm{m}$ ("active LDs") (Figure 6). This feature may contribute to explain the toxic effects of saturated FA, like PA, on hepatocytes.

The effect of $\mathrm{OA}$ promoting bigger and more abundant LDs compared to PA (Figure 1) (5) has also been observed in other cell types, such as pancreatic $\beta$-cells (38), chondrocytes (39), and H9C2 cardiomyoblasts (40). This greater capacity of $\mathrm{OA}$ to be esterified to TG and accumulated into LDs likely explains its reduced lipotoxicity compared to PA. Furthermore, OA reportedly ameliorates PA-induced toxicity in hepatocytes $(5,7,41)$, although it is unclear whether OA promotion of PA redirection to TG is the primary protective mechanism (6). For instance, $\mathrm{OA}$ also induces the redistribution of ceramide synthases to LDs. These enzymes catalyze the storage of ceramide as acylceramide, thereby decreasing the toxic effect of ceramide accumulation (42).

LDs participate in controlling energy metabolism and thus communicate with other organelles, relying on regions of close contact, including mitochondria, ER, and peroxisomes (9). The LD-mitochondria association has been reported particularly in tissues with a high TG oxidation and storage capacity, such as liver, heart, brown adipose tissue, and skeletal muscle $(8,13,30)$. Notably, starvation-induced LDs in cardiomyocytes require a fused mitochondrial network to ensure mitochondrial oxidation of FA (43), suggesting that LD and mitochondrial dynamics and function are highly interrelated. Our study, like others, shows that PA triggers rapid mitochondrial fragmentation. On the other hand, OA treatment induced a slower increase in mitochondrial fragmentation, which was not as marked as the one induced by PA (Figure 2). These results support the idea that saturated and unsaturated FA differentially affect the mitochondrial fusion/fission equilibrium.

LD-mitochondria physical contacts are observable from yeast to mammalian cells (9), and their extent varies according to lipolytic stimuli $(17,44)$, exercise (45) or starvation (43, 46). Under starvation conditions, LD-mitochondria contacts increase, which supports FA transfer to mitochondria for $\beta$-oxidation (43). Similar results were observed in brown adipocytes, where cold exposure increased LD-mitochondria interaction, supporting thermogenesis $(14,40)$. More recently, Benador et al. reported in brown adipocytes that mitochondria in close contact with LDs maintain their oxidative capacity but have low levels of FA oxidation, thereby supporting LD growth by providing ATP for TG synthesis (8). Hence, two models of LD-mitochondria coupling have been so far described: the interaction that favors LD consumption and energy production, and the interaction that prompts LD expansion $(30,47)$. In the hepatocyte in vitro model presented here, we found that OA promotes a steeper increase in LD-mitochondria contacts than PA (Figure 2), suggesting that in HepG2 cells, OA feeding evokes the type of LD-mitochondria that mediates LD expansion.

Remarkably, only OA treatment increased PLIN5 and MNF2 protein levels, which putatively support the observed increment in LD-mitochondria contacts (Figure 3). Reportedly, PLIN5 is present in multiple cells and tissues, including hepatic and heart muscle cells (48). In accordance with our results, hepatocytes from liver-specific PLIN5 KO mice display fewer LD-mitochondria contacts, reduced hepatic TG synthesis and FA oxidation, and are more susceptible to develop hepatic insulin resistance (49). On the contrary, mice overexpressing liverspecific PLIN5 fed with a high-fat diet exhibit severe steatosis without worsening glucose homeostasis. Surprisingly, these animals have lower fasting insulin levels, suggesting preservation of insulin sensitivity (50). Similar to PLIN5, MFN2 is needed for LD-mitochondria interaction, as shown in brown adipose tissue, where it supports FA-fueled thermogenesis (51). Accordingly, MFN2 ablation protects against high-fat diet-induced insulin resistance in brown adipose tissue (17). On the other hand, in our study model, PA treatment only raised PLIN2, but not PLIN5 nor MFN2 protein levels. This observation agrees with another study showing that PLIN2 promotes liver steatosis in mice (52). However, how PLIN2 participates in LD-mitochondria coupling remains to be unveiled. Altogether, our observations indicate that $\mathrm{OA}$ and PA treatments induce different sets of LDmitochondria tethers, which underlie the distinctive functional coupling between both organelles.

Additionally, our study showed that PA treatment triggers a faster and more pronounced mitochondrial fragmentation than OA, concomitant with markedly higher OCR, $\Delta \psi \mathrm{m}$ and ATP levels (Figures 2, 4). These results support the idea that saturated and unsaturated fatty acids differentially regulate the mitochondrial fusion/fission equilibrium and bioenergetics, generating two mitochondrial networks with distinct metabolic profiles. This agrees with the study of Benador et al. in brown adipocytes, in which smaller mitochondria contributed to fatty acid oxidation more actively, while larger mitochondria mainly 


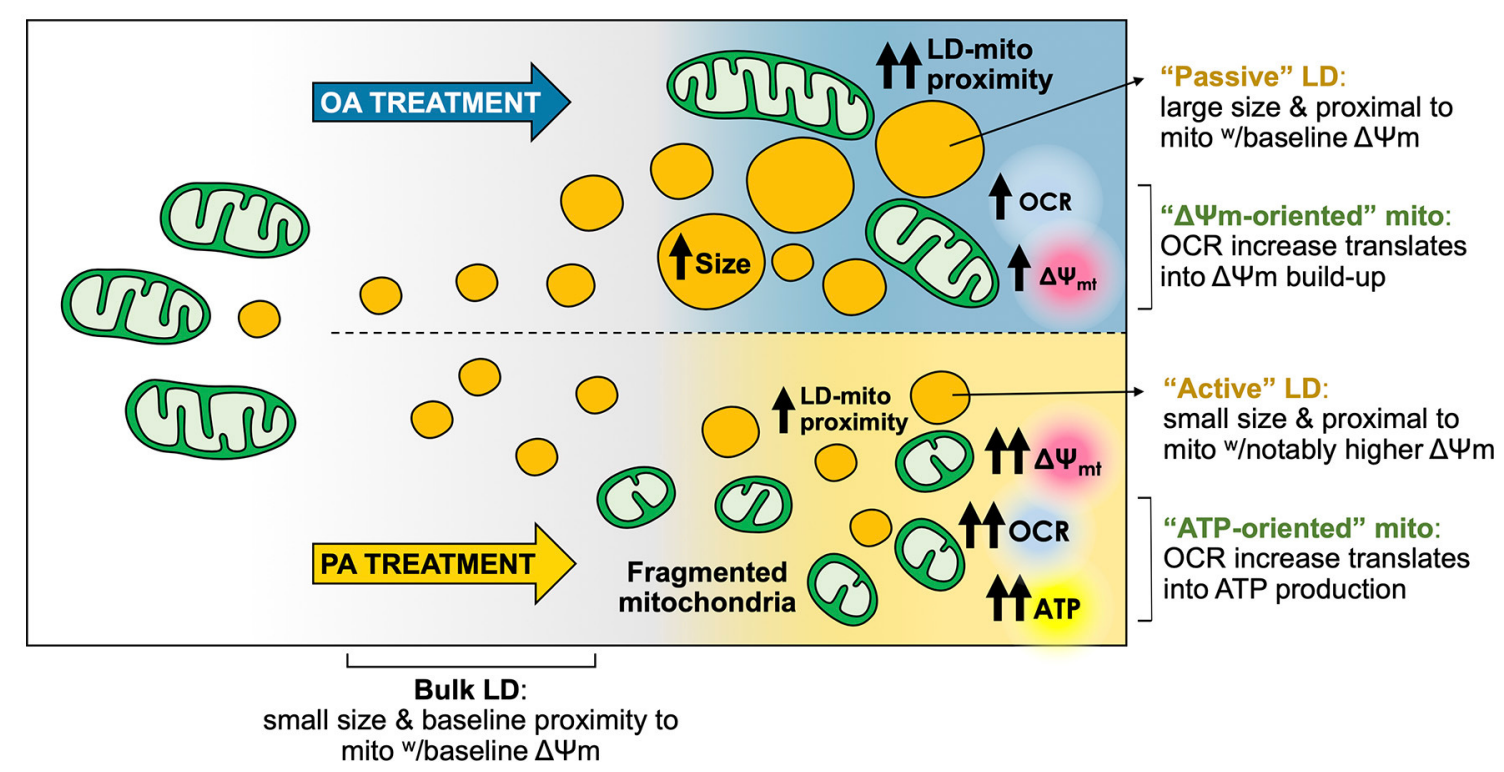

FIGURE 6 | Oleic and palmitic acids favor different lipid droplet and mitochondria sub-populations with distinct morphological and bioenergetic profiles in HepG2 cells. Both oleic acid (OA) and palmitic acid (PA) induce the formation of lipid droplets (LDs) in HepG2 cells. OA induces a steep nucleation process, followed by a steady growth of LDs. PA stimulates a moderate increase in the number of LDs, which maintain a small size. Moreover, while OA does not affect mitochondrial morphology, PA elicits fragmentation of the mitochondrial network. Both treatments boost mitochondrial respiration (OCR), but with different outcomes. OA mainly heightens the overall transmembrane potential of the mitochondrial network ( $\Delta \psi \mathrm{m}$-oriented network). On the other hand, PA treatment favors ATP generation (ATP-oriented network). LD-mitochondria physical proximity increases upon treatment with either fatty acid. OA evokes higher levels of LD-mitochondria interaction compared to PA. However, LDs from OA-treated cells are rather "passive", as their larger size associates with lower- $\Delta \psi m$ mitochondria, compared to PA. LDs from PA-treated cells are apparently more "active", as they are smaller and interact with higher- $\Delta \psi \mathrm{m}$ mitochondria, which is concomitant with increased ATP levels.

participated in LD growth by providing ATP for the synthesis of TG (8). These divergent mitochondrial behaviors explain not only the differences in LD morphology after PA and OA treatments but also different metabolic parameters (Figure 4F): PA oxidation greatly fuels OCR and ATP production (i.e., ATP-oriented mitochondrial network), while OA accumulation induces a limited increase in OCR and ATP, leading to $\Delta \psi \mathrm{m}$ accretion (i.e., $\Delta \psi \mathrm{m}$-oriented mitochondria network). These dissimilar metabolic fates partially explain the higher toxicity of saturated FA in hepatocytes compared to unsaturated FA.

Consistent with our aforementioned interpretation, the analysis of LD-mitochondria proximity showed that OAinduced $\mathrm{LD}$ is more extensively proximal to mitochondria than PA (Figure 5). However, these mitochondria maintain a baseline metabolic activity, as indicated by MitoTracker Orange staining. Thus, we hypothesized that OA-induced LD does not fuel oxidative metabolism, but instead consumes ATP, thus acting as "passive" LDs in terms of mitochondrial bioenergetics. Interestingly, LD-bound mitochondria under OA treatment displayed a lower $\Delta \psi \mathrm{m}$ compared to the rest of the mitochondrial network. This can be explained by the fact that the ATP consumption required for LD growth contributes to the dissipation of the $\Delta \psi \mathrm{m}$. On the other hand, PA promoted the accumulation of small-sized LDs proximal to mitochondria with higher $\Delta \psi \mathrm{m}$ (Figure 5). Accordingly, we speculate that these LDs fuel mitochondrial bioenergetics, thus acting as metabolically "active" LDs that do not particularly consume ATP, thus leading to $\Delta \psi \mathrm{m}$ accumulation in the mitochondria that stand nearer.

Reportedly, mitochondria associate with LDs in tissues exhibiting a high capacity for TG storage and oxidation, such as the liver, heart, brown adipose tissue, and skeletal muscle $(8,13$, 30). Notably, contrasting evidence has shown that mitochondrial oxidation of LD-derived fatty acids requires mitochondria fusion in MEF cells (43) or fission in brown adipocytes (8), suggesting that LD metabolism and mitochondrial dynamics are interrelated, and seemingly dependent on the cell type. Moreover, our study also showed that the fatty acid type also determines the $\mathrm{LD} /$ mitochondrial dynamics ratio.

\section{CONCLUSION}

In sum, our data uncover two patterns of LD-mitochondria interaction in response to treatment with two different fatty acids. On the one hand, OA led to triglyceride accumulation, concomitant with increased LD-mitochondria proximity. Under this condition, the overall mitochondrial network underwent only a slight metabolic boost, which mainly exerted a $\Delta \psi \mathrm{m}$ oriented role instead of contributing to ATP production. Meanwhile, OA-induced LD appeared rather "passive", precluding fatty acids from mitochondrial oxidation, thereby thwarting the bioenergetic boost of nearby mitochondria. On the other hand, PA-induced a slight increase in LD accumulation. Newly formed LDs were seemingly "active" and associated with 
mitochondria with higher OCR and $\Delta \psi \mathrm{m}$, compared to the rest of the network (Figure 6). Thus, we hypothesized that PA treatment renders mitochondria more ATP-oriented compared to OA treatment, due to higher substrate availability. Our results underscore the importance of the dietary FA composition in the development of NAFLD, where saturated FA promote hepatic steatosis with mitochondrial dysfunction, which in turn can promote NAFLD progression.

\section{DATA AVAILABILITY STATEMENT}

The raw data supporting the conclusions of this article will be made available by the authors, without undue reservation.

\section{AUTHOR CONTRIBUTIONS}

$\mathrm{RT}$ and VP conceived and designed the study. AE, FD-C, and JB performed the experiments. AE, RB-S, and VP analyzed the data. AE, VP, and RT interpreted the data. AE, RB-S, VP, and

\section{REFERENCES}

1. Younossi Z, Tacke F, Arrese M, Chander Sharma B, Mostafa I, Bugianesi E, et al. Global perspectives on nonalcoholic fatty liver disease and nonalcoholic steatohepatitis: hepatology. Hepatology. (2019) 69:2672-82. doi: 10.1002/hep.30251

2. Arrese M, Barrera F, Triantafilo N, Arab JP. Concurrent nonalcoholic fatty liver disease and type 2 diabetes: diagnostic and therapeutic considerations. Expert Rev Gastroenterol Hepatol. (2019) 13:849-66. doi: 10.1080/17474124.2019.1649981

3. Meex RCR, Blaak EE. Mitochondrial dysfunction is a key pathway that links saturated fat intake to the development and progression of NAFLD. Mol Nutr Food Res. (2021) 65:1900942. doi: 10.1002/mnfr.201900942

4. Burlingame B, Nishida C, Uauy R, Weisell R. Fats and fatty acids in human nutrition. Ann Nutr Metab. (2009) 55:5-300. doi: 10.1159/000228993

5. Ricchi M, Odoardi MR, Carulli L, Anzivino C, Ballestri S, Pinetti A, et al. Differential effect of oleic and palmitic acid on lipid accumulation and apoptosis in cultured hepatocytes. J Gastroenterol Hepatol. (2009) 24:83040. doi: 10.1111/j.1440-1746.2008.05733.x

6. Chen X, Li L, Liu X, Luo R, Liao G, Li L, et al. Oleic acid protects saturated fatty acid mediated lipotoxicity in hepatocytes and rat of non-alcoholic steatohepatitis. Life Sci. (2018) 203:291-304. doi: 10.1016/j.lfs.2018.04.022

7. Moravcová A, Cervinková Z, Kučera O, Mezera V, Rychtrmoc D, Lotková H. The effect of oleic and palmitic acid on induction of steatosis and cytotoxicity on rat hepatocytes in primary culture. Physiol Res. (2015) 64:S627-636. doi: 10.33549/physiolres.933224

8. Benador IY, Veliova M, Mahdaviani K, Petcherski A, Wikstrom JD, Assali EA, et al. Mitochondria bound to lipid droplets have unique bioenergetics, composition, and dynamics that support lipid droplet expansion. Cell Metab. (2018) 27:869-85.e6. doi: 10.1016/j.cmet.2018.03.003

9. Olzmann JA, Carvalho P. Dynamics and functions of lipid droplets. Nat Rev Mol Cell Biol. (2019) 20:137-55. doi: 10.1038/s41580-018-0085-z

10. Wei Y, Rector RS, Thyfault JP, Ibdah JA. Nonalcoholic fatty liver disease and mitochondrial dysfunction. World J Gastroenterol. (2008) 14:1939. doi: 10.3748/wjg.14.193

11. Eisner V, Picard M, Hajnóczky G. Mitochondrial dynamics in adaptive and maladaptive cellular stress responses. Nat Cell Biol. (2018) 20:75565. doi: 10.1038/s41556-018-0133-0

12. Aon MA, Bhatt N, Cortassa SC. Mitochondrial and cellular mechanisms for managing lipid excess. Front Physiol. (2014) 5:282. doi: $10.3389 /$ fphys.2014.00282
RT drafted the manuscript reviewed by all authors. All authors contributed to the article and approved the submitted version.

\section{FUNDING}

This work was supported by grants from the Agencia Nacional de Investigación y Desarrollo (ANID), Chile: FONDECYT 1191078 to RT; 1190743 to VP; 11201267 to RB-S; FONDAP 15130011 to RT, VP, and RB-S; PAI Insertion Program 77170004 to RB-S; University of Chile U-inicia UI-006/19 to RB-S and U-redes 2018-G_2018-35 to VP; and CRP-ICGEB CHL18-04 to VP.

\section{ACKNOWLEDGMENTS}

We would like to thank Sebastián Leiva for his excellent technical assistance, and Dr. Ana María Avalos for proofreading and revising our manuscript.

13. Wang H, Sreenivasan U, Hu H, Saladino A, Polster BM, Lund LM, et al. Perilipin 5, a lipid droplet-associated protein, provides physical and metabolic linkage to mitochondria. J Lipid Res. (2011) 52:215968. doi: 10.1194/jlr.M017939

14. Wang H, Sreenivasan U, Gong D-W, O'Connell KA, Dabkowski ER, Hecker PA, et al. Cardiomyocyte-specific perilipin 5 overexpression leads to myocardial steatosis and modest cardiac dysfunction. J Lipid Res. (2013) 54:953-65. doi: 10.1194/jlr.M032466

15. Freyre CAC, Rauher PC, Ejsing CS, Klemm RW. MIGA2 links mitochondria, the ER, and lipid droplets and promotes de novo lipogenesis in adipocytes. Mol Cell. (2019) 76:811-25.e14. doi: 10.1016/j.molcel.2019.09.011

16. Pidoux G, Witczak O, Jarnæss E, Myrvold L, Urlaub H, Stokka AJ, et al. Optic atrophy 1 is an A-kinase anchoring protein on lipid droplets that mediates adrenergic control of lipolysis. EMBO J. (2011) 30:437186. doi: 10.1038/emboj.2011.365

17. Boutant M, Kulkarni SS, Joffraud M, Ratajczak J, Valera-Alberni M, Combe R, et al. Mfn2 is critical for brown adipose tissue thermogenic function. $E M B O J$ (2017) 36:1543-58. doi: 10.15252/embj.201694914

18. García-Rúa V, Feijóo-Bandín S, Rodríguez-Penas D, Mosquera-Leal A, AbuAssi E, Beiras A, et al. Endolysosomal two-pore channels regulate autophagy in cardiomyocytes. J Physiol. (2016) 594:3061-77. doi: 10.1113/JP271332

19. Parra V, Moraga F, Kuzmicic J, López-Crisosto C, Troncoso R, Torrealba N, et al. Calcium and mitochondrial metabolism in ceramide-induced cardiomyocyte death. Biochim Biophys Acta. (2013) 1832:1334-44. doi: 10.1016/j.bbadis.2013.04.009

20. Bravo-Sagua R, López-Crisosto C, Parra V, Rodriguez-Peña M, Rothermel BA, Quest AFG, et al. mTORC1 inhibitor rapamycin and ER stressor tunicamycin induce differential patterns of ER-mitochondria coupling. Sci Rep. (2016) 6:36394. doi: 10.1038/srep36394

21. Parra V, Verdejo HE, Iglewski M, Del Campo A, Troncoso R, Jones D, et al. Insulin stimulates mitochondrial fusion and function in cardiomyocytes via the Akt-mTOR-NFкB-Opa-1 signaling pathway. Diabetes. (2014) 63:7588. doi: $10.2337 / \mathrm{db} 13-0340$

22. Bravo-Sagua R, Parra V, Ortiz-Sandoval C, Navarro-Marquez M, Rodríguez AE, Diaz-Valdivia N, et al. Caveolin-1 impairs PKA-DRP1-mediated remodelling of ER-mitochondria communication during the early phase of ER stress. Cell Death Differ. (2019) 26:1195-212. doi: 10.1038/s41418-018-0197-1

23. Parra V, Altamirano F, Hernández-Fuentes CP, Tong D, Kyrychenko V, Rotter D, et al. Down syndrome critical Region 1 Gene, Rcan1, helps maintain a more fused mitochondrial network. Circ Res. (2018) 122:e2033. doi: 10.1161/CIRCRESAHA.117.311522 
24. Li F, Munsey TS, Sivaprasadarao A. TRPM2-mediated rise in mitochondrial $\mathrm{Zn} 2+$ promotes palmitate-induced mitochondrial fission and pancreatic $\beta$-cell death in rodents. Cell Death Differ. (2017) 24:1999-2012. doi: 10.1038/cdd.2017.118

25. Li J, Wang Y, Wang Y, Wen X, Ma X-N, Chen W, et al. Pharmacological activation of AMPK prevents Drp1-mediated mitochondrial fission and alleviates endoplasmic reticulum stress-associated endothelial dysfunction. $J$ Mol Cell Cardiol. (2015) 86:62-74. doi: 10.1016/j.yjmcc.2015.07.010

26. Kuzmicic J, Parra V, Verdejo HE, López-Crisosto C, Chiong M, García L, et al. Trimetazidine prevents palmitate-induced mitochondrial fission and dysfunction in cultured cardiomyocytes. Biochem Pharmacol. (2014) 91:32336. doi: 10.1016/j.bcp.2014.07.022

27. Manders EMM, Verbeek FJ, Aten JA. Measurement of co-localization of objects in dual-colour confocal images. J Microsc. (1993) 169:37582. doi: 10.1111/j.1365-2818.1993.tb03313.x

28. Cohen S. Lipid droplets as organelles. Int Rev Cell Mol Biol. (2018) 337:83110. doi: 10.1016/bs.ircmb.2017.12.007

29. Henne WM, Reese ML, Goodman JM. The assembly of lipid droplets and their roles in challenged cells. EMBO J. (2018) 37:e98947. doi: 10.15252/embj.201898947

30. Benador IY, Veliova M, Liesa M, Shirihai OS. Mitochondria bound to lipid droplets: where mitochondrial dynamics regulate lipid storage and utilization. Cell Metab. (2019) 29:827-35. doi: 10.1016/j.cmet.2019.02.011

31. Parra V, Verdejo H, del Campo A, Pennanen C, Kuzmicic J, Iglewski $\mathrm{M}$, et al. The complex interplay between mitochondrial dynamics and cardiac metabolism. J Bioenerg Biomembr. (2011) 43:47-51. doi: 10.1007/s10863-011-9332-0

32. Vásquez-Trincado C, García-Carvajal I, Pennanen C, Parra V, Hill JA, Rothermel BA, et al. Mitochondrial dynamics, mitophagy and cardiovascular disease. J Physiol. (2016) 594:509-25. doi: 10.1113/JP271301

33. Scheideler M, Herzig S. Let's burn whatever you have: mitofusin 2 metabolically re-wires brown adipose tissue. EMBO Rep. (2017) 18:103940. doi: 10.15252/embr.201744341

34. Brand MD, Nicholls DG. Assessing mitochondrial dysfunction in cells. Biochem J. (2011) 435:297-312. doi: 10.1042/BJ20110162

35. Ferree A, Shirihai O. Mitochondrial dynamics: the intersection of form and function. Adv Exp Med Biol. (2012) 748:1340. doi: 10.1007/978-1-4614-3573-0_2

36. Herker E, Vieyres G, Beller M, Krahmer N, Bohnert M. Lipid droplet contact sites in health and disease. Trends Cell Biol. (2021) 31:34558. doi: 10.1016/j.tcb.2021.01.004

37. Haslam DW. James WPT obesity. Lancet. (2005) 366:1197209. doi: 10.1016/S0140-6736(05)67483-1

38. Cnop M, Hannaert JC, Hoorens A, Eizirik DL, Pipeleers DG. Inverse relationship between cytotoxicity of free fatty acids in pancreatic islet cells and cellular triglyceride accumulation. Diabetes. (2001) 50:17717. doi: 10.2337/diabetes.50.8.1771

39. Vázquez-Mosquera ME, Fernández-Moreno M, Cortés-Pereira E, Relaño $\mathrm{S}$, Dalmao-Fernández A, Ramos-Louro $\mathrm{P}$, et al. Oleate prevents palmitateinduced mitochondrial dysfunction in chondrocytes. Front Physiol. (2021) 12:670753. doi: 10.3389/fphys.2021.670753

40. Akoumi A, Haffar T, Mousterji M, Kiss RS, Bousette N. Palmitate mediated diacylglycerol accumulation causes endoplasmic reticulum stress, Plin2 degradation, and cell death in H9C2 cardiomyoblasts. Exp Cell Res. (2017) 354:85-94. doi: 10.1016/j.yexcr.2017.03.032

41. Listenberger LL, Han X, Lewis SE, Cases S, Farese RV, Ory DS, et al. Triglyceride accumulation protects against fatty acid-induced lipotoxicity. Proc Natl Acad Sci U S A. (2003) 100:3077-82. doi: 10.1073/pnas.0630588100

42. Senkal CE, Salama MF, Snider AJ, Allopenna JJ, Rana NA, Koller A, et al. Ceramide Is Metabolized to Acylceramide and Stored in Lipid Droplets. Cell Metab. (2017) 25:686-97. doi: 10.1016/j.cmet.2017.02.010
43. Rambold AS, Cohen S, Lippincott-Schwartz J. Fatty Acid Trafficking in Starved Cells: Regulation by Lipid Droplet Lipolysis, Autophagy, and Mitochondrial Fusion Dynamics. Dev Cell. (2015) 32:678-92. doi: 10.1016/j.devcel.2015.01.029

44. Yu J, Zhang S, Cui L, Wang W, Na H, Zhu X, et al. Lipid droplet remodeling and interaction with mitochondria in mouse brown adipose tissue during cold treatment. Biochimica et Biophysica Acta (BBA) - Molec Cell Res. (2015) 1853:918-28. doi: 10.1016/j.bbamcr.2015. 01.020

45. Tarnopolsky MA, Rennie CD, Robertshaw HA, Fedak-Tarnopolsky SN, Devries MC, Hamadeh MJ. Influence of endurance exercise training and sex on intramyocellular lipid and mitochondrial ultrastructure, substrate use, and mitochondrial enzyme activity. Am J Physiol Regul Integr Comp Physiol. (2007) 292:R1271-1278. doi: 10.1152/ajpregu.0047 2.2006

46. Herms A, Bosch M, Reddy BJN, Schieber NL, Fajardo A, Rupérez $\mathrm{C}$, et al. AMPK activation promotes lipid droplet dispersion on detyrosinated microtubules to increase mitochondrial fatty acid oxidation. Nat Commun. (2015) 6:7176. doi: 10.1038/ncomm s8176

47. Bosch M, Parton RG, Pol A. Lipid droplets, bioenergetic fluxes, and metabolic flexibility. Semin Cell Dev Biol. (2020) 108:33-46. doi: 10.1016/j.semcdb.2020.02.010

48. Mason RR, Watt MJ. Unraveling the roles of PLIN5: linking cell biology to physiology. Trend Endocrinol Metabol. (2015) 26:144-52. doi: 10.1016/j.tem.2015. 01.005

49. Keenan SN, Meex RC, Lo JCY, Ryan A, Nie S, Montgomery MK, et al. Perilipin 5 deletion in hepatocytes remodels lipid metabolism and causes hepatic insulin resistance in mice. Diabetes. (2019) 68:543-55. doi: 10.2337/db18-0670

50. Trevino MB, Mazur-Hart D, Machida Y, King T, Nadler J, Galkina $\mathrm{EV}$, et al. Liver perilipin 5 expression worsens hepatosteatosis but not insulin resistance in high fat-fed mice. Mol Endocrinol. (2015) 29:141425. doi: 10.1210/me.2015-1069

51. Mahdaviani K, Benador IY, Su S, Gharakhanian RA, Stiles L, Trudeau $\mathrm{KM}$, et al. Mfn2 deletion in brown adipose tissue protects from insulin resistance and impairs thermogenesis. EMBO Rep. (2017) 18:112338. doi: 10.15252/embr.201643827

52. Orlicky DJ, Libby AE, Bales ES, McMahan RH, Monks J, La Rosa FG, et al. Perilipin-2 promotes obesity and progressive fatty liver disease in mice through mechanistically distinct hepatocyte and extra-hepatocyte actions. $J$ Physiol. (2019) 597:1565-84. doi: 10.1113/JP277140

Conflict of Interest: The authors declare that the research was conducted in the absence of any commercial or financial relationships that could be construed as a potential conflict of interest.

Publisher's Note: All claims expressed in this article are solely those of the authors and do not necessarily represent those of their affiliated organizations, or those of the publisher, the editors and the reviewers. Any product that may be evaluated in this article, or claim that may be made by its manufacturer, is not guaranteed or endorsed by the publisher.

Copyright (C) 2021 Eynaudi, Díaz-Castro, Bórquez, Bravo-Sagua, Parra and Troncoso. This is an open-access article distributed under the terms of the Creative Commons Attribution License (CC BY). The use, distribution or reproduction in other forums is permitted, provided the original author(s) and the copyright owner(s) are credited and that the original publication in this journal is cited, in accordance with accepted academic practice. No use, distribution or reproduction is permitted which does not comply with these terms. 